\title{
Supporting interdisciplinary careers for sustainability
}

\author{
Universities and research centres around the world have made significant progress towards establishing \\ collaborative, interdisciplinary initiatives in sustainability science. However, more needs to be done to support the \\ career development of junior sustainability scholars whose work is often team based and outreach oriented.
}

\section{J. Nicolas Hernandez-Aguilera, Weston Anderson, Allison L. Bridges, M. Pilar Fernandez, Winslow D. Hansen, Megan L. Maurer, Elisabeth K. Ilboudo Nébié and Andy Stock}

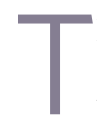

he overwhelming events of 2020 , including the increasingly frequent wildfires, the COVID-19 outbreak and the social unrest ignited by systemic exclusion and racism, have illustrated, repeatedly, that solutions to sustainability crises require unravelling complex interactions that do not fit neatly into a single discipline. To take just one example, wildfires in Australia, Western Europe, and North and South America present an emergency resulting from decades of sprawling urban growth and climate change. Addressing such sustainability crises requires actionable interdisciplinary science based on a foundation of partnership among academics and broader society. Given the extensive work on how universities can produce scholarship to meaningfully address those sustainability challenges, we ask: do current career paths and structures incentivize and facilitate the required collaboration?

Diverse authors have made grand calls for change in academic institutions to support impactful sustainability science $^{1-3}$. As a global group of early-career sustainability scholars, working at a leading interdisciplinary research institute, we recognize that the support we have received in the form of postdoctoral fellowships, seed funding and mentored guidance is the result of this effort. However, we also recognize practical and institutional barriers that, from our perspective, discourage bright individuals with diverse backgrounds from pursuing a career in the sustainability space. A clear career path for interdisciplinary early-career scientists has not developed in parallel to the value that universities ascribe to real-world problems and the proliferation and effort of sustainability research centres. Moreover, many of the skills that make scholars competitive in academic and non-academic positions for sustainability, such as public communication skills, community relationship building, policy-oriented writing and the open-mindedness needed for collaboration beyond their disciplines, are undervalued in academia. An inherent bias remains in the system towards singular disciplinary expertise and publications, even in universities with interdisciplinary centres. Overall, the academic community frequently sends a clear message: interdisciplinary work is important, but do not expect the system to easily recognize $\mathrm{it}^{2,4}$.

The traditional criteria for judging candidates for faculty positions and the promotion of junior faculty continue to be out of sync with the timing, funds and communication channels required for interdisciplinary research ${ }^{1-3,5,6}$. Specifically, community-based research or even purely academic interdisciplinary collaborations demand substantial time commitments ${ }^{7}$, but the promotion clock is inflexible. Early-career researchers, whose success is judged heavily on publications and grant awards, are forced to consider the trade-offs of preparing and coordinating higher-risk, team-based, interdisciplinary papers and proposals ${ }^{8}$, versus narrowly focused grants and publications. Besides, peer reviewers sometimes perceive that interdisciplinary manuscripts do not contribute enough to a specific field or are unfamiliar with other disciplines' methods and approaches?. Moreover, sustainability scholars' impact is usually measured by the number of times their papers are cited and does not consider how effectively this research connects with a global audience beyond academics. As a result, the advance of interdisciplinary careers has been a slow process through institutions designed around strict disciplinary research structures and incentives ${ }^{1,2,6,10}$.

\section{Moving forward}

Academia needs to embrace all levels of disciplinary integration to provide sustainable answers. For instance, in the case of the COVID-19 pandemic response, specialized scholars rely on basic science to develop vaccines, while others collaborate to understand the biophysical and socio-economic factors that lead to potential public health threats. Simultaneously, other researchers can coordinate the best responses with local and regional health authorities. This case reflects an approach that requires flexibility in the application of traditional, discipline-specific performance metrics on those scholars interested in collaborating to solve real-world problems without penalizing the performance of scholars conducting basic research. Not all researchers should be measured with the same metrics and the academy should be able to find and adopt the hiring and tenure policies required to facilitate the coexistence of all levels of disciplinary integration, from intradisciplinary and basic science, to inter- and transdisciplinary approaches that combine and apply knowledge production and engage non-academic partners.

Universities and research centres need to better define and communicate their expectations for interdisciplinary researchers and the standards to which they will be held for finding and securing permanent positions. We advocate for the creation of truly interdisciplinary professor positions and the elimination of hosting departments in the hiring and performance evaluation for these positions. Interdisciplinary research centres that attract experts and practitioners of diverse backgrounds have played a central role in scientific innovation for decades. For instance, some of the greatest technological and scientific advancements of the late twentieth century came from initiatives that bridged the gap between laboratory research and operations. The Bell Labs and MIT Building 20 in the USA, and the Fraunhofer Institutes in Germany were behind important discoveries in a wide range of areas including telecommunications, digital technologies and linguistics; similar collaborative breakthroughs are needed now. 
Structural changes also require shifts in the norms and culture that underpin the hiring and promotion of interdisciplinary positions in academia. Specifically, schools should weigh a more comprehensive portfolio and alternative metrics that demonstrate candidates' capacities for collaborative goal-setting, communication skills and collaboration with diverse stakeholders $^{11}$. It is evident that classical bibliometrics used to measure scholarly performance are insufficient when attempting to measure how successfully scholars influence media and policy outcomes. Furthermore, all metrics can be biased, gamed and may reproduce existing injustices. For example, female researchers face higher risks of online abuse than men, which discourages social media activities ${ }^{12}$ and hence can bias metrics of social media influence against women. Briefs or reports that are highly influential for policy may never be published and activities such as disseminating research results to local interest groups are difficult to quantify. Thus, we call for a broad definition of productivity in sustainability research, using a diverse set of metrics to overcome the limitations associated with each, and openly communicated standards for how different kinds of impact will be weighted in hiring and promotions. Such metrics should include traditional products such as academic papers or grants, but equally, consider science-based policy briefs, influence on programmes and planning, evidence of successful community engagement and town-hall gatherings, demonstrated socio-environmental problem solving, experience leading diverse interdisciplinary teams and evidence of engagement. The Committee on Institutional Cooperation (now the Big Ten Academic Alliance) was an early leader in the promotion and use of tenure metrics for public outreach ${ }^{13}$. Drawing from their experience, a consortium of sustainability schools could work to promote the adoption of clearly defined hiring and tenure-track promotion systems based on new metrics of both interdisciplinary and public engagement.

Universities are not the only institutions that have the power to ensure interdisciplinary schools are built. Public and private funding institutions, such as the UK's Research Excellence Framework, the Australian Council of Learned Academies and the Inter-American Institute for Global Change Research, also incentivize universities to transform traditional structures by providing large-scale solutions grants with longer time frames, tailored calls and innovative ways to assess the current and potential impact of interdisciplinary projects $^{14}$. Moreover, there is precedent for national-level initiatives targeting global reforms across the university system. In the nineteenth century, and in response to the industrial revolution, the US land-grant university (LGU) model endowed higher education centres that focused on practical agriculture, engineering and science ${ }^{2}$. Although aspects of the LGU are problematic (that is, land expropriation ${ }^{15}$ ), in the twenty-first century, a similarly grand-scale programme could support an agenda for sustainability and interdisciplinarity, inside and outside the USA (for example, Colombia-LGU consortium for agriculture and peacebuilding). Lastly, publishers can promote a more transparent review process for interdisciplinary research, reducing unjustified biases and increasing confidence among authors. For example, establishing inter-reviewer discussions (co-review) and certifying the effort invested by reviewers in the process. Co-review contributions could be considered by schools as an objective metric of scholar recognition and commitment to promote interdisciplinary research.

Interdisciplinarity has proven advantageous for scholars who find themselves at innovative institutions that recognize the value of collaborative work. It is encouraging to observe the increasing number of universities that have strengthened cross-cutting research centres, provided seed funding to early-career interdisciplinary teams and appointed professional officers of research at those centres. For instance, the Earth Institute at Columbia University recently announced a Climate School. Arizona State University Global Futures Lab, in alliance with the University of Washington and the Nippon Foundation, announced a centre focused on social equity and ocean sustainability. Such initiatives are uniquely positioned to include profound transformations in career development and capitalize on new funding streams such as the National Science Foundation Convergence, Navigating the New Arctic and the Dynamics of Socio-Environmental Systems grants, which support proposals that combine natural environment and social systems topics. The time is long past due, however, for expanding institutional support and recognizing the value of interdisciplinary scholars and scholarships.

Sustainability crises threaten human well-being at local to global scales. A new era of actionable interdisciplinary science is needed to develop impactful solutions, now. While some encouraging steps have been taken to engender a rich field of sustainability science in academia, many of the changes will be moot if talented and creative scholars choose 'safer' and better-established career trajectories. As early-career scholars with global expertise in solutions-oriented sustainability science, we feel that swift changes in the structure of universities and their hiring and promotion strategies are critical, if we are to address the world's most pressing social and environmental problems before it is too late. Implementing some of our recommendations may not be easy. It will require investment, will and creativity. But if not now, then when?

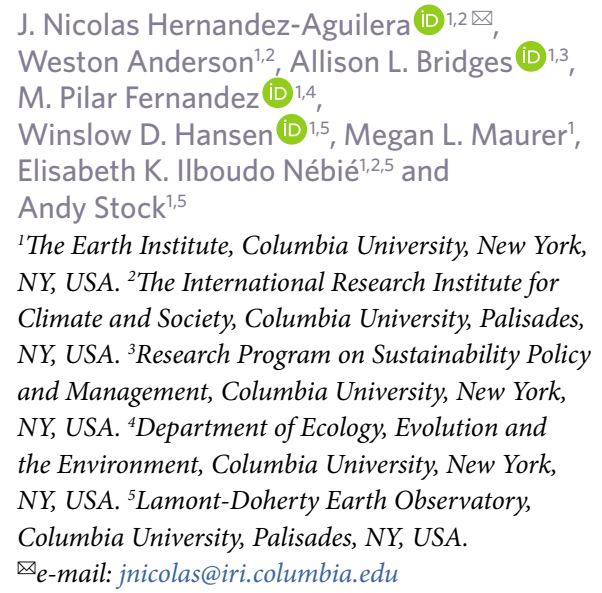

Published online: 28 January 2021 https://doi.org/10.1038/s41893-020-00679-y

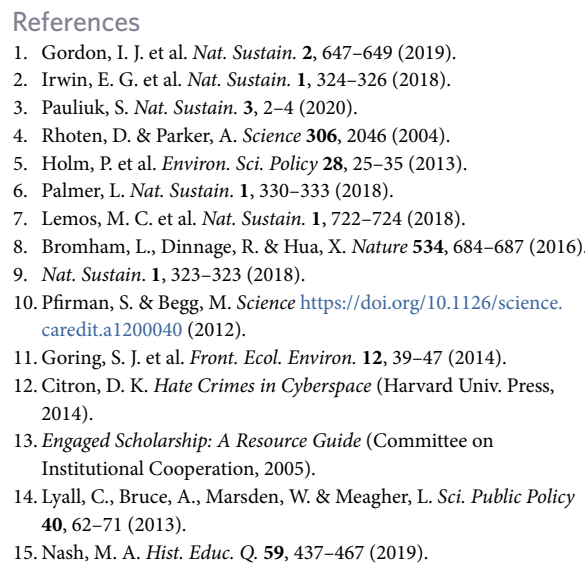

Competing interests

The authors declare no competing interests.

\section{Additional information}

Peer review information Nature Sustainability thanks Carina Wyborn and the other, anonymous, reviewer(s) for their contribution to the peer review of this work. 